\title{
Postoperative complications of surgery in the sellar region: about 79 cases
}

A.Naili, H.Triki, S.Bouderra, Intensive care unit -EPH Sidi-Ghiles-Tipaza-Algeria

\section{Introduction:}

The Tumors of the sellar region represent $10 \%$ of the intracranial tumors, they are generally benign but considered as malignant by their location and their post operative complications.

Our objective is to establish the rate of postoperative complications after surgery of the sellar region in our department, and to propose some rules to follow in order to prevent and minimize the occurrence of such complications.

\section{Patients and method:}

79 patients scheduled for neurosurgical procedures were included in the retrospective study over one year period (from June 1st, 2016 to September 10, 2017). We collected clinical, etiological and therapeutic data of those with postoperative complications.

\section{Discussion :}

*The hormonal imbalance was noted $48 \mathrm{~h}-72 \mathrm{~h}$ post operative.

*Diabetes insipidus occurred at 2sd day, transient (craniopharyngioma). *Hydroelectrolytic disorder at 10th day *Infectious complications occurred late beyond 15 days.

*The detection of leakage of the cerebrospinal fluid intraoperatively is essential in the prevention of postoperative rhinorrhea, The number of meningitis on fistula in the CSF has decreased thanks to the established rules for the fight against nosocomial infections.

-Must Correct endocrine disorders before surgery within a few weeks by prescribing the drug blocker: prolactin, $\mathrm{GH}, \mathrm{ACTH}$ to reduce the volume of the tumor and facilitate excision.

*Systematic prevention of adrenal insufficiency by a HHC substitution pattern, pre and postoperative.
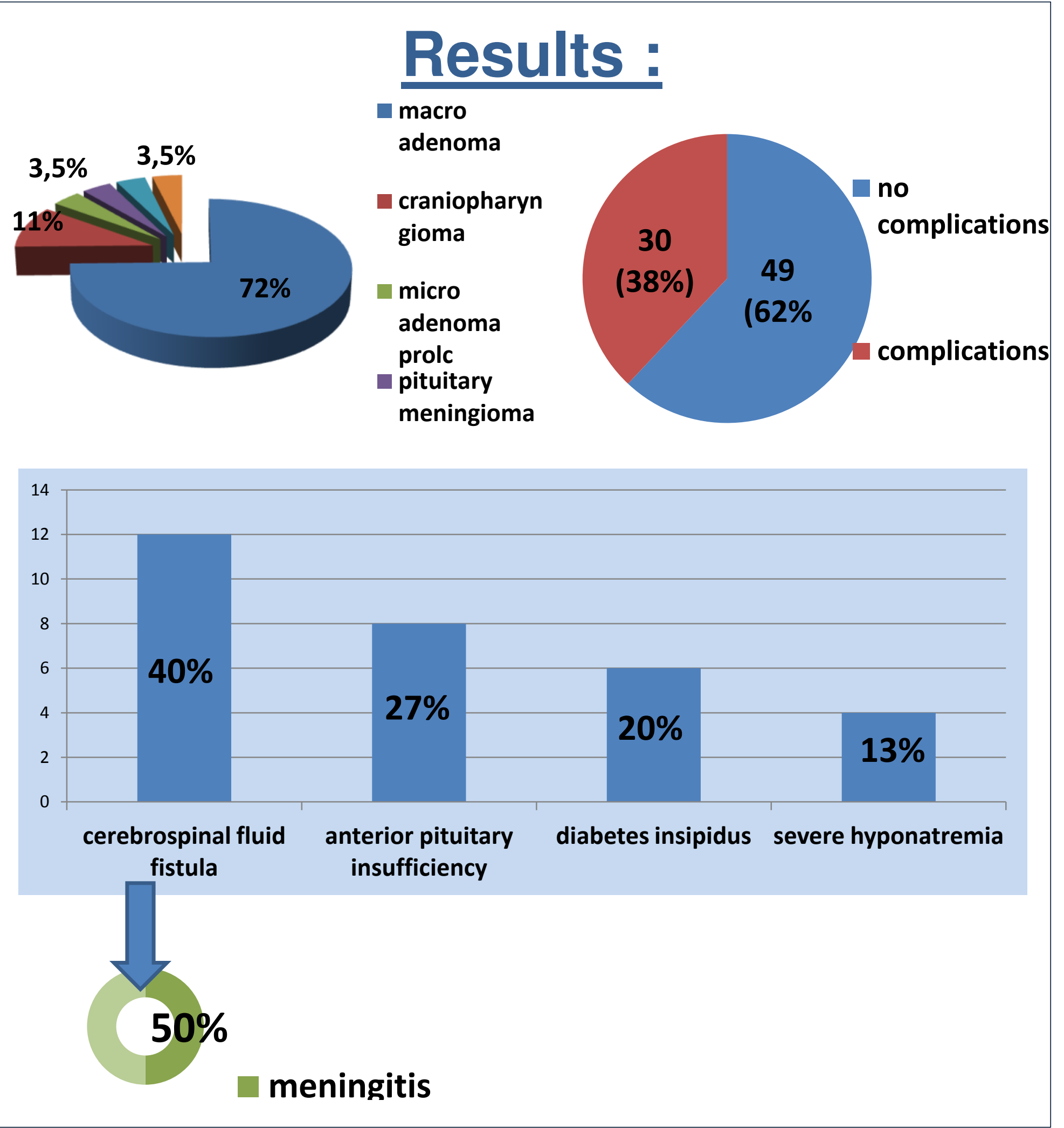

\section{Conclusion :}

*Surgery in the sellar region carries various postoperative risks, one of which may be fatal.

*Medical and anesthetic management remain challenges for the resuscitator,

which begin preoperatively with a good evaluation and continue through the per and postoperative period. .

*Management requires a multidisciplinary approach, perfect knowledge of the complications of this surgery, immediate and remote postoperative biological and neurological monitoring.

${ }^{*}$ A service protocol must be established to properly support this type of surgery Interest to push the searches on it.

2-Perioperative Management of Patients Undergoing Transsphenoidal Pituitary Surgery journal of saoudit anesthesia Nemergut, Edward C. MD*+; Dumont, Aaron S. MD+; Barry, Usha T. MD*; Laws, Edward R. MD+ 2005

3- Pituitary Surgery: Systematic Review and 3 Case Presentations Toron Keenan Neurosurgery, Li Ka Shing Knowledge Institute, St. Michael's Hospital, Toronto, Ontario, Canada

4- Persky MS, Brunner E, Copper PR, Cohen NL. Perioperative complication of transsphenoidal excision for pituitary adenomas. Skull Base Surgery. 1996;6:231-5. [PMC free article] [PubMed] 5-Anaesthesia and pituitary disease, Anaesthesia, Critical Care \& Pain | Volume 11 Number 42011 the British Journal of Anaesthesia

6-Complications of transsphenoidal surgery in patients with pituitary adenoma: experience at a single centre 